\title{
IoT Technology Based Smart Systems Control Methodology Using Net Pi Network Platform
}

\author{
Ayad Ghany Ismaeel, Omar Farooq Rafat, Sarmad Nozad Mahmood \\ \{ayad.ghany@uoalkitab.edu.iq, om.faruk1983@gmail.com, sarmadnmahmood@gmail.com\} \\ Department of Computer Technical Engineering, Alkitab University, Iraq
}

\begin{abstract}
The IoT technological advances amazed the world and offered the smart house networks a perfect opportunity to release such advancements. This article illustrates a groundbreaking design and construction of a low-cost IoT based house system automation. The mechanization of the network is seen as a major challenge, particularly outside the building. The proposed device regulates and tracks the house condition at any time and in worldwide remotely based on NET PI network platform using ESP8266 node MCU microcontroller. The ESP8266 functions as an access point AP, connected to the network across the Wi-Fi station (router). The smart house system based on the webserver acts under the guidance of the Net Pie server showing the output results of the sensors. Furthermore, the webserver helps the registered owner to switch house devices ON and OFF remotely. Finally, the article confirms that the NETPI web server eliminates the restriction available on the other servers.
\end{abstract}

Keywords: ESP8266 Microcontroller, Smart House System, Modular Control System, Temperature and Humidity Sensor, Gas Detection System, Net Pi Network Platform.

\section{Introduction}

The Internet of Things (IoT) is a collection of electronic, mechanical and non-electronic devices capable of being remotely configured, operated and controlled over communication networks. The person who is in charge can control local and external equipment and can turn on / off devices, move trip circuits between multiple devices, access devices, remote controls, etc [1]. If a person feels very uncomfortable due to the weather outside when the person goes home, a fairly useful program can be used. The user may switch on the air conditioning to cool the space depending on the position in which the user is in relation to his house and his house's ambient temperature. Another scenario is when the customer cannot plan something to eat right after they arrive home $[1,2]$. Furthermore, before the person arrives home, the oven or coffee maker can be switched $\mathrm{ON}$ and controlled depending on the position of the user and the temperature of the appliance. The operation of electrical or electronic devices can then be configured and controlled via the IoT via actuators [3]. In light of this, there exist several issues concerning IoT that should be stated as follows:

1. Several controllers are needed in many situations, one for each system that must be managed in the same Graphical User Interface GUI.

2. Implementation of a portal capable of controlling all sensors and sensor systems with different brands and communication protocols [4].

3. Discussion on positioning the execution of the mechanism in the Gateway, the sensor/device itself or the cloud [5]. 
Both systems of IoT technology act synchronously with the absence of the customer, delivering accurate and reliable performance. This research aims to create an IoT-based solution within the smart house system parameters via a mechanical actuator ON / OFF remote control system. The proposed system incorporates the advancements of the ESP8266 NodeMCU platform and employs the NET PI web server program as a configurable device, cloud service, sensor, and actuator controller. Thus it is low-cost, open-source hardware and software-based solution. During its growth, the IoT design was used and this allows the device, as part of a Smart Home, a high degree of modularization thus enabling it to be developed in the future. The approach was tested via a case study performed at Alkitab University.

\section{Literature Survey and Motivation}

The IoT uses a wide variety of devices, protocols, technologies, networks, middleware, applications, systems, and data, all forming a heterogeneous network. This will increase the degree of interoperability and complexity. Because of this situation, several groups such as ITU, ETSI, OpenIoT are developing interoperability standards and IoT protocols, among others. However, the high fragmentation and development of vertical IoT systems have increased in a multi-standard context, where features, functions and devices are combined [6]. In [7] an energy- and position-based IoT system is proposed controlled in networks, which is also based on a smartphone and cloud computing platform. This provides energy efficiency in buildings and organizations, as a complete system on a large scale. The problem with this proposal is that it is difficult and costly to implement, so it is not recommended to be applied in an ordinary smart house. A conceptual IoT device called AAL-IoTSys, which includes a Smart Gateway as a key component is proposed in [6]. This enables many heterogeneous devices to be interoperable across different networks, protocols and technologies based on (eg WiFi, ZigBee, Bluetooth, IEEE 802.15.4, 6 LowPAN). The main motivation behind this work is to design an advanced modular control system to monitor and interact with human instructions remotely. Several sensors are employed to satisfy smart house system requirements such as measuring the temperature, humidity, LPG gas modifications, heating, ventilation, and air conditioning (HVAC) systems and storing the energy in the batteries through PV panels. To address our problem, the NETPI architecture can be the best choice for fulfilling the requirements as long as we want to use more than one microcontroller in a single GUI inside our program.

\section{IoT System Components}

The proposed IoT system is constructed from several elements configured and connected with respect to the connection with the employed microcontroller. These elements and sensors are identified as follows:

\subsection{ESP8266 Node MCU}

ESP8266 Node MCU is a new aspect of microcontroller developed by Arduino Corporation. This microcontroller acts as Arduino microcontroller specifications regardless of AVR processors $[8,9]$ which lead to Arduino IDE $\mathrm{C}++$ compilers to compile the entire package. Due to the functionality introduced by the ESP team, the package is considered a complete kit to minimize the individual sectors that needed to be connected to the board for specific roles to be performed. The word 'Core' was provided to the community of software units that are 
required by using MCU language to compile the Arduino $\mathrm{C}++$ headers. Compared to the design methodology which formed Arduino core under the hegemony of ESP8266 Wi - Fi centered on GitHub ESP8266 core website, the ingenuity of the ESP8266 module leads to the construction of robust and complete systems. This module is a machine learning framework that integrates firmware between ESP8266 and NodeMCU. The MCU module shown in Figure 1 runs under 802.11n and 802.11b Networks oversight. This ensures that it can be used concurrently as an Access Point AP, Wi-Fi device or both of them together [10].

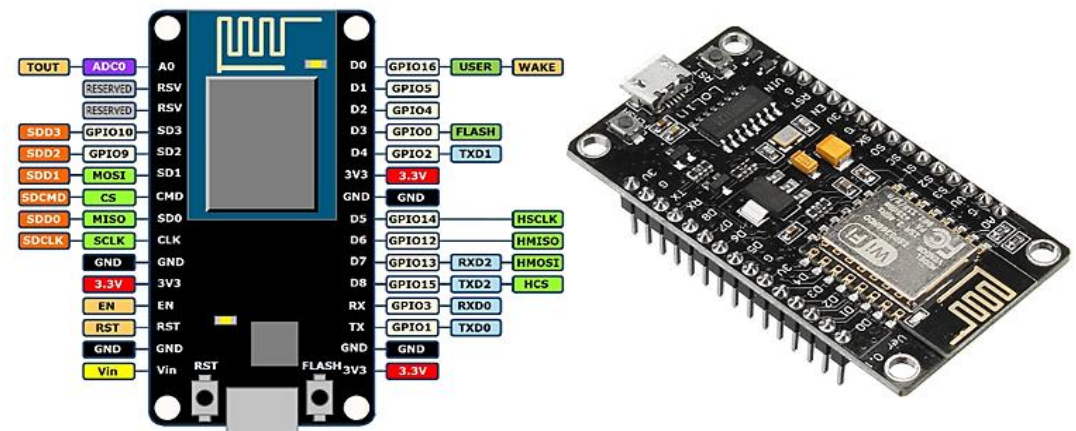

Fig.1. ESP8266 NodeMCU Microcontroller

\subsection{Temperature - Humidity Sensor}

In a single distinctive form, the Temperature - Humidity sensor known as DHT11 reads and monitors temperature and humidity degrees. The DHT11 sensor that presents the Temperature (T) and Humidity $(\mathrm{H})$ is handled with optical signal output adjustment in a complex manner. Due to the private digital signal processing in the sensing equipment, the sensor guarantees remarkable sensitivity and excellent long term stability. This module includes a resistive humidity subsystem and an NTC temperature panel, attached to a high-performance 8-bit microcontroller, providing excellent quality, fast response, anti-interference functionality and cost-effectiveness $[11,9]$. In this work, the DHT sensor checks both $(\mathrm{T})$ and $(\mathrm{H})$ and passes the readings on to the Net Pie website via the ESP8266 board. The element is composed of three terminals the Vcc, Data, and Gnd. The sensor shows marvels superiority if it is attached to a microcontroller's digital pins. As shown in Figure 3 [12,13], the VCC pin must be supplied by $5 \mathrm{~V}$ of ESP8266 MCU, the data is chosen to be attached to ESP8266's optical pin GPIO2 (D4), and the sensor's Gnd is linked to the ESP8266 board's Gnd pin.

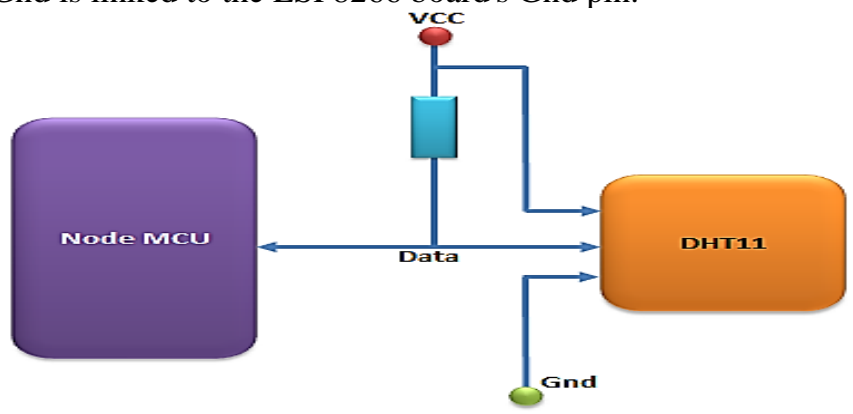

Fig.2. Temperature and Humidity DHT11 Sensor 


\subsection{Liquefied Petroleum Gas (LPG) Sensor}

The SnO2-based Liquefied Petroleum Gas (LPG) exhibits mild conductivity in the clean air. The sensor in Figure 3 states that in case of higher gas content, the conductivity will be directly proportional in such a way that it gets higher. For propane, LPG, gasoline, carbon, and other steams the output of the MQ-2 sensor is deemed extremely greater. However, the module's cost limitations are ideal for specific applications [14, 15]. The sensor discovers the existence of the flammable gas because of the elevation of temperature performed by the heating components inside the module. Once gas leakage is observed the sensor's conductivity increases proportionally with gas concentration as a working theory. The VH heater voltage provides the sensor with working temperature; however, the loop voltage VC determines the voltage over the load resistance VRL [9].

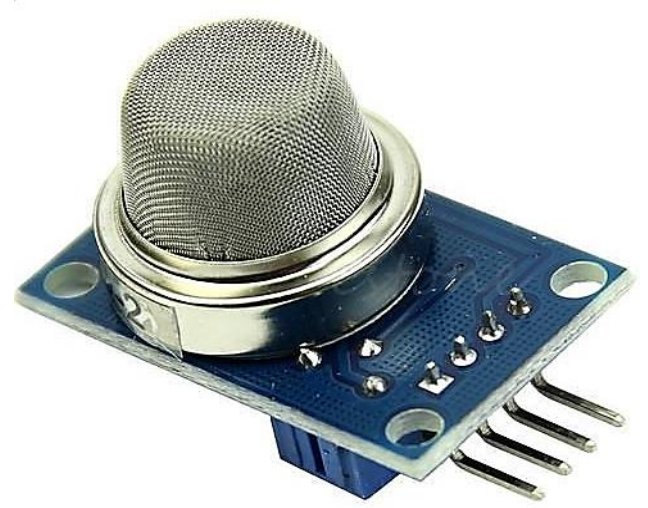

Fig.3. Temperature and Humidity DHT11 Sensor

The efficiency of the proposed sensor can be increased by using a reasonable RL to fulfil the following equation $[16,9]$ :

$\mathrm{P}_{\mathrm{S}}=\frac{V c^{2} * R_{S}}{\left(R_{S}+R_{L}\right)^{2}}$

Where,

$P_{s}:$ Sensitive power of the body.

$V_{c}$ : Loop voltage.

$R_{S}$ : Sensing resistance.

$R_{L}$ : Load resistance.

Furthermore, the suggested LPG kit is perfectly suitable for the discovery of gas leaks and the quality of the air depending on the following set of equations:

Gas Leak $=$ Concetratio $\leq$ Gas Spread $\leq 102$ Air Quality $=$ Gas leak -1023

There, the value 1023 reflects the full analog reading range about the matching state between the gas sensor and the node MCU board. The meaning of load resistance makes no sense to be conditionally restricted particularly when the output voltage is supposed to be measured via the 
load resistance RL around the point at the source and the Gnd terminal as shown in Figure 4. Conversely, it is preferable to choose the value of RL around $2-47 \mathrm{~K} \Omega$, which means that the lower value offers less sensitivity, the higher value offers less precision.

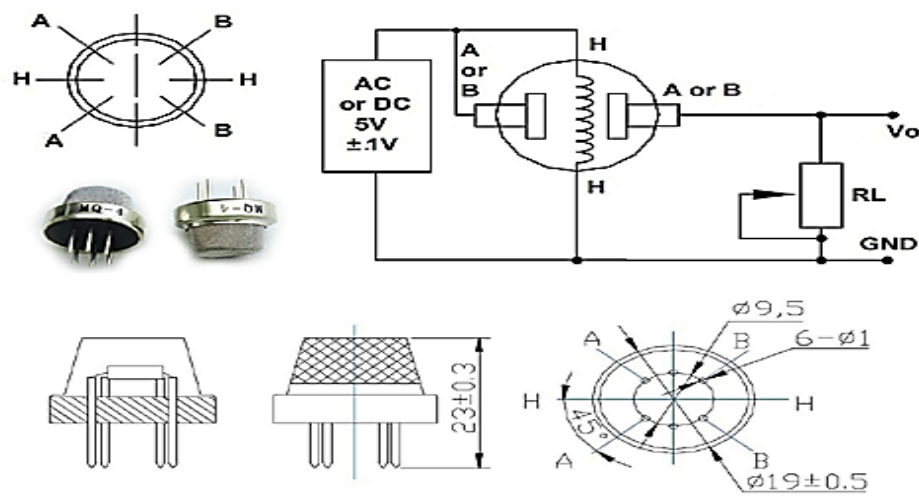

Fig.4. Interior design of LPG sensor in general

Certainly, the sensor for air concentration of 1000 ppm - LPG or butane can be adjusted by specifying the load resistance value $\mathrm{RL}=20 \mathrm{~K} \Omega$ [9].

\section{Solar Energy and Connection Methodology}

The amount of energy is considered solar energy when produced in the form of heat and radiation [17]. The sun radiates both light and heat as a natural source of energy using a number of ever-changing developments such as thermal technology, construction, heating, molten salt, power plant, and artificial respiration. The vast amount of solar power available renders energy a very promising supply [18]. Approximately, Thirty percent of the solar radiation escapes to space and the remainders are consumed by oceans, dust and landmasses. PV cells Transform power from sunlight to direct current (DC). The voltage regulator acts as regulation of the solar panel power that is redirected back to the solar panel causing serious battery corruption. The battery system works like electric power storage during the night when the sunlight is not accessible as exposed in Figure 5. Normally, the huge applications need to employ an inverter device in order to convert the stored sunlight from DC into AC high voltage. However, this work represents only the impression of solar cell construction and due to cost limitations, the investor is not allowed in this system [5]. 


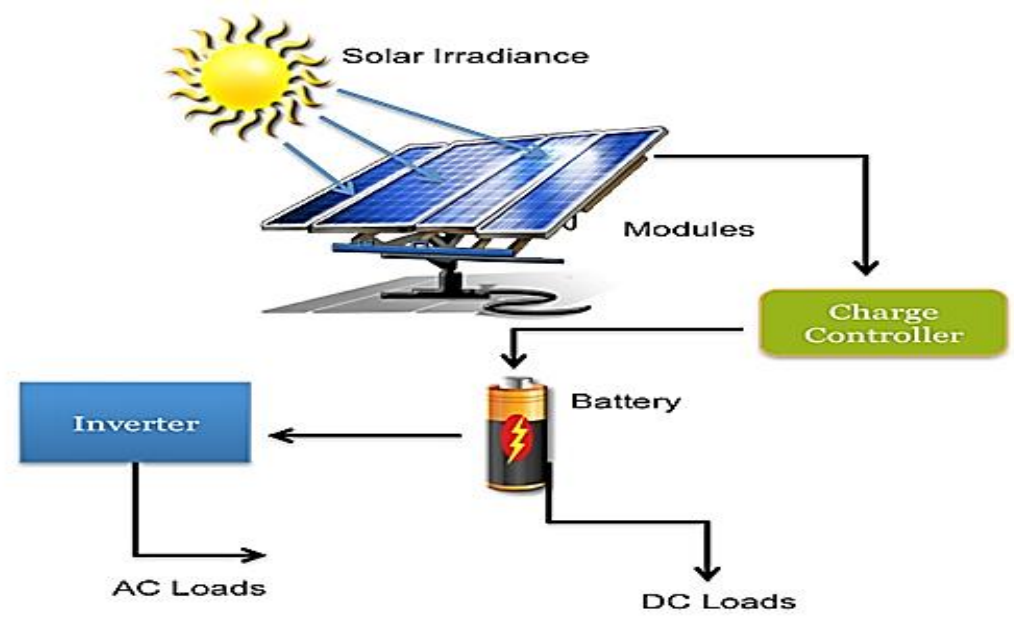

Fig.5. Solar Panel Connection Methodology

Based on the semiconductor materials, the cells transform solar radiation directly into electricity which is divided into positive and negative areas. This technique is often applied to build low-cost solar cells, as well as high efficiency in conversion. Once the cell collects the photons from sunlight, the electrons are kicked free of silicon atoms and pulled off by a conductor grid, then the electrical current will be generated and pass due to the pressure. A PV panel composed of solar cell circuits enclosed in an eco-protective laminate which are the main features of the PV network. In this project, the employed PV provides the following specifications:

Peak power $=3 \mathrm{~W}$ power.

Maximum power current $(\operatorname{Imp})=0.34 \mathrm{~A}$.

Open circuit voltage $(\mathrm{Voc})=12 \mathrm{~V}$.

It is worth mentioning that the battery system that employed in this project is a lithium-ion battery limited by $12 \mathrm{~V}, 1.2 \mathrm{~A}$, stand by use $(13.5-13.8) \mathrm{V}$ and cycle use $(14.4-15) \mathrm{V}$. In order to amplify the current, we intended to connect four PV panels in parallel as shown in Figure 6 to get the following specifications:

$$
\begin{gathered}
\text { for } P V \ldots \\
4 P V * I_{m p}=1.36 \mathrm{~A} \\
4 P V * \text { Peak pwer }=12 \mathrm{~W} \\
\text { for battery system } \ldots \\
P=I * V \\
1.2 * 12=14.4 \mathrm{~W}
\end{gathered}
$$




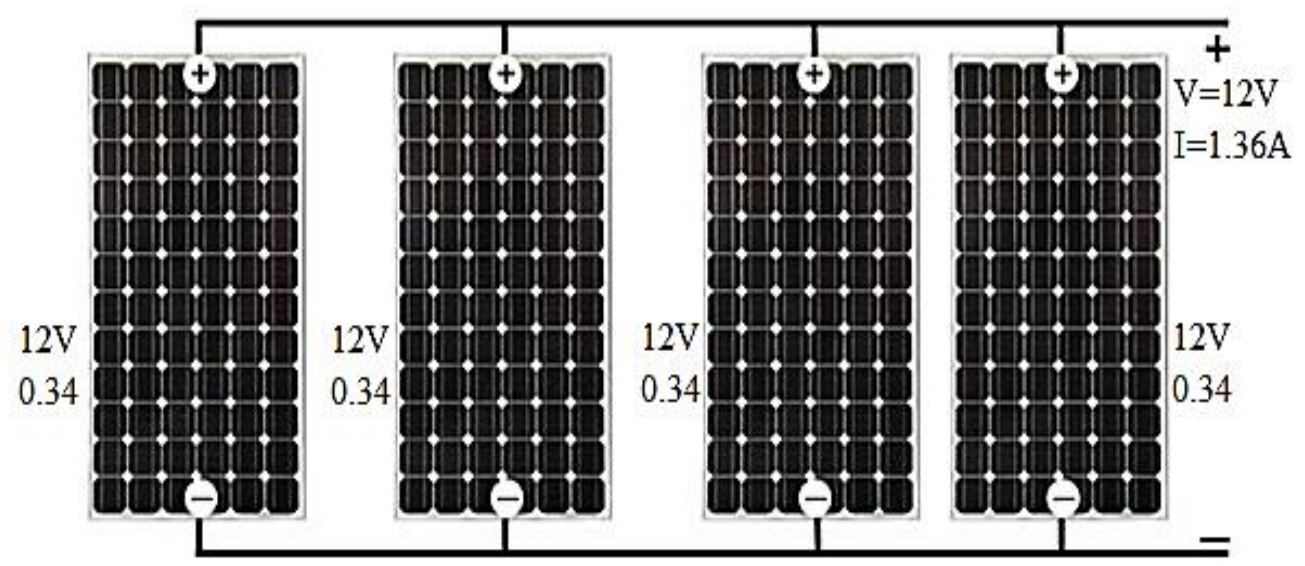

Fig.6. Solar Panels Connection in Parallel

As stated in equation (2), the power needed to charge the battery is 14.4 .W. However, the PV panels give maximally around $12 \mathrm{~W}$. This matter is not a big problem because in the worst cases the charging time of the battery system $(\tau)$ can barely take a little more time than usual to fully charge the battery. The remaining IoT system parts that are connected as exposed in Figure 7 can be modelled such that they fulfil the desired environment specifications.

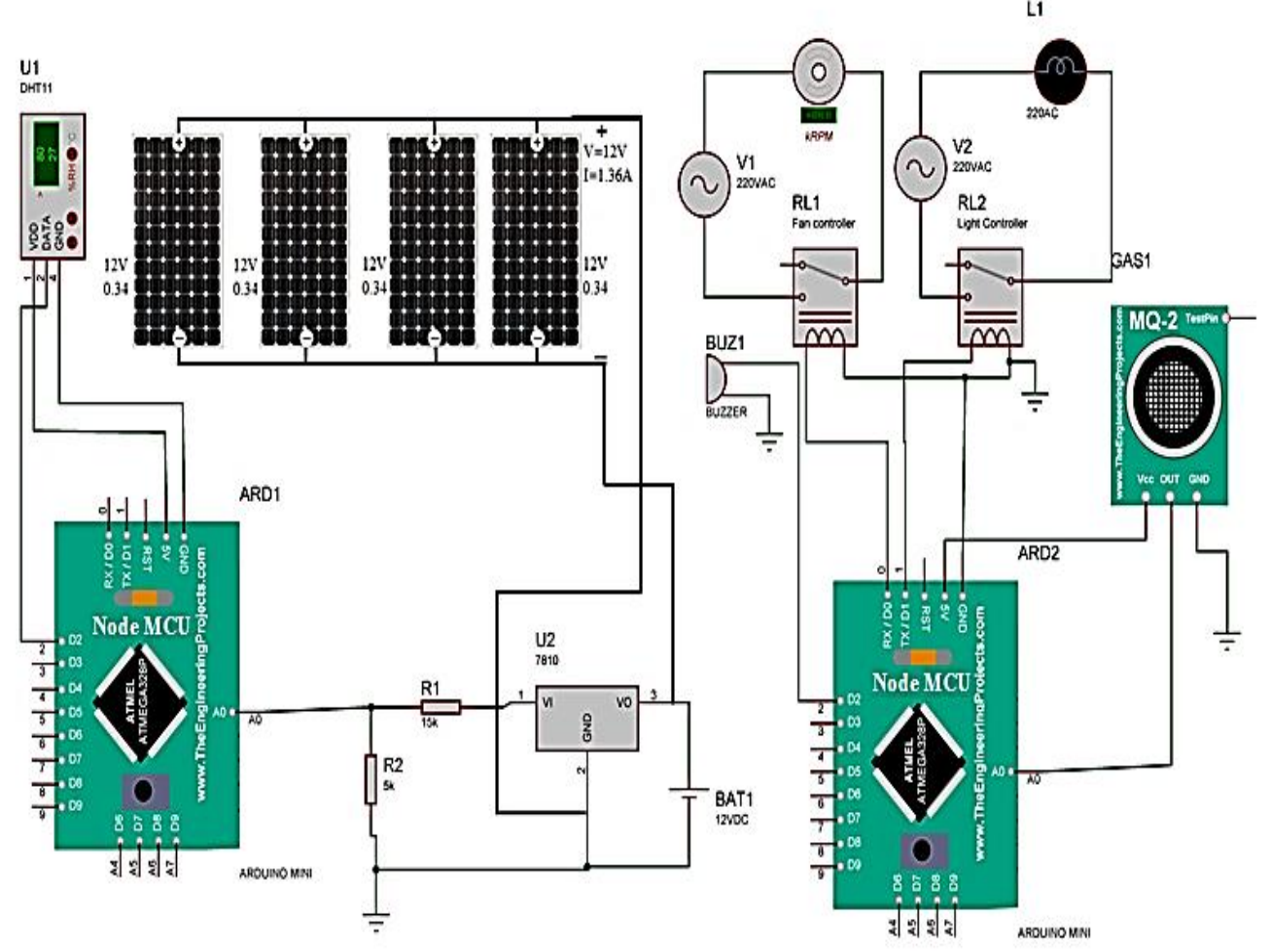

Fig.7. Overall System Connection 
The DHT11 sensor monitors the changes in the temperature and humidity at the proposed location and in addition to the solar panel in order to measure sun radiation accordingly. Furthermore, the sensor used to measure gas concentration (MQ2) transfers the variations in the quality of the air in between $(0-1023)$ analog reads in order to unveil any gas leak available in the system. The main important problem stated above is that the capability of using more than one microcontroller to control more devices (in a single and unique GUI) as performed in the project via NET PI network platform (https://netpie.io/), unlike the other servers violated this assumption. As a result and for more clarity, the flowchart $[9,16,19]$ in Figure 8 describes the scenario of the monitoring by reading the sensors simultaneously to take an optimum control action remotely.

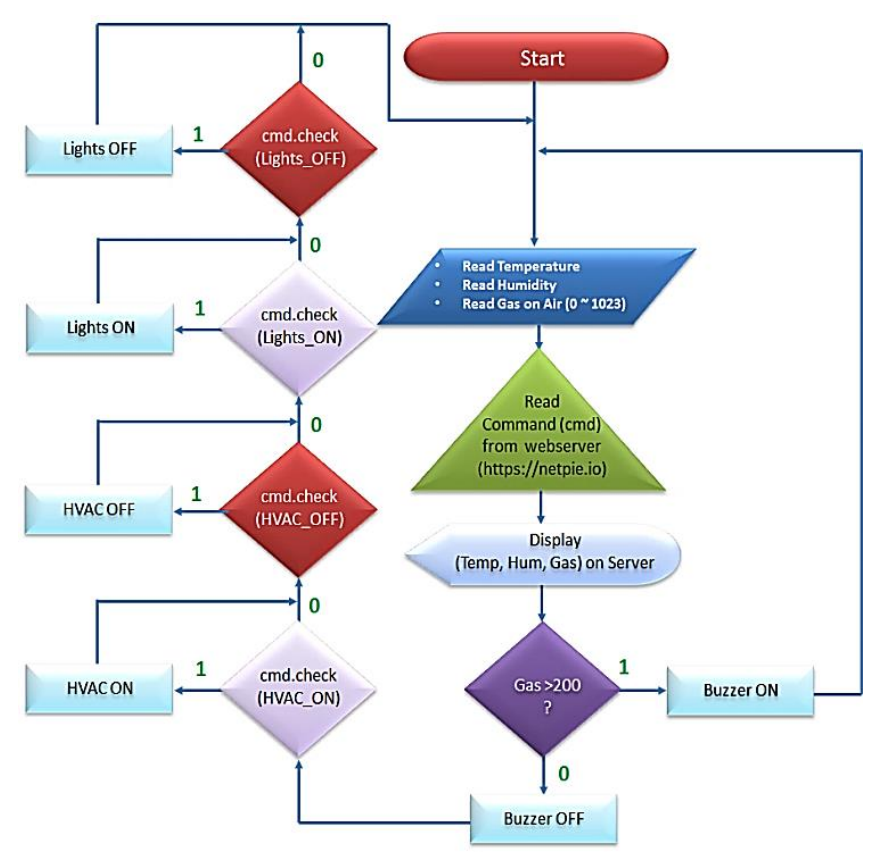

Fig.8. Working Principle as Flowchart

The term HVAC refers to Heating, ventilation, and air conditioning systems (represented by the FAN in this work) or even the lighting system in the house that both are needed to be controlled either manually or automatically depending on some conditions. The control system design to dominate such a challenge is considered strictly critical particularly when we deal with high AC voltages $(220 \mathrm{~V})$. However, the motivation of this project has been fulfilled successfully using ESP8266 microcontroller based NET PI web server control system as shown in Figure 9. 


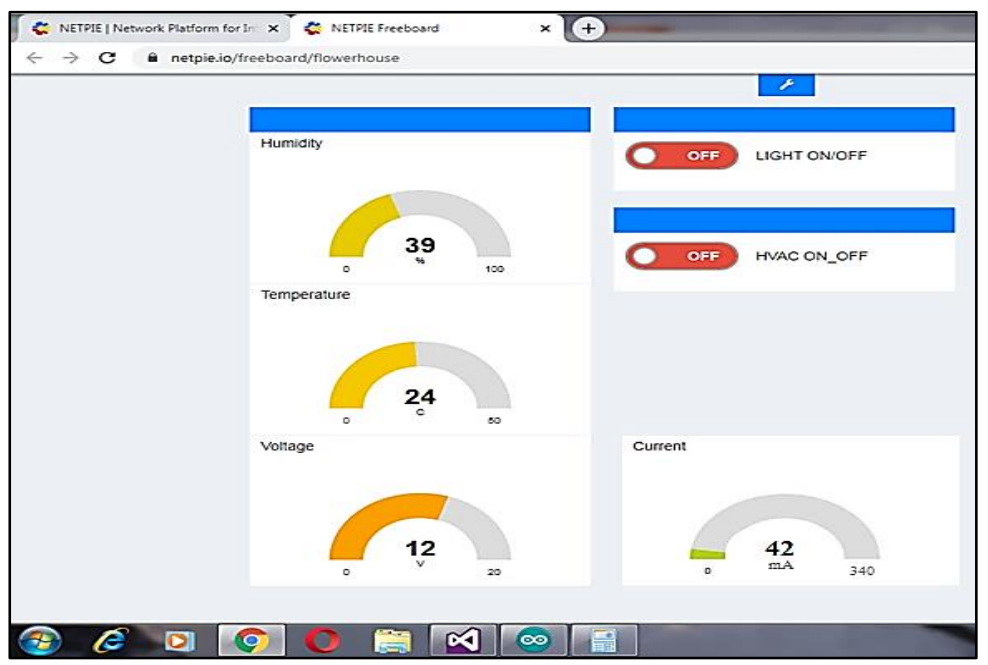

Fig.9. Real Time NETPI Network Platform

Figure 9 gives evidence that NET PI can merge several microcontrollers in the same GUI. The LPG gas sensor and the Voltage / Current indicators both are connected to the analog terminal of the microcontroller, knowing that the node MCU controllers have only one analog pin. Hereby, this can present a clear indication that we are controlling two-node MCU microcontrollers in a single GUI site (objective is approved). Furthermore, node MCU microcontroller deals only with $3.3 \mathrm{~V}$ as an output voltage. Hence, the most important question is: (how to read the $12 \mathrm{~V}$ battery voltage through the analog pin of the node MCU?). This mater needs to apply matching conditions using the fundamental of voltage divider rule scheme by connecting the voltage terminals with respect to the resistances R1 and R2 as described wisely in Figure 10 and the equations on the right side.

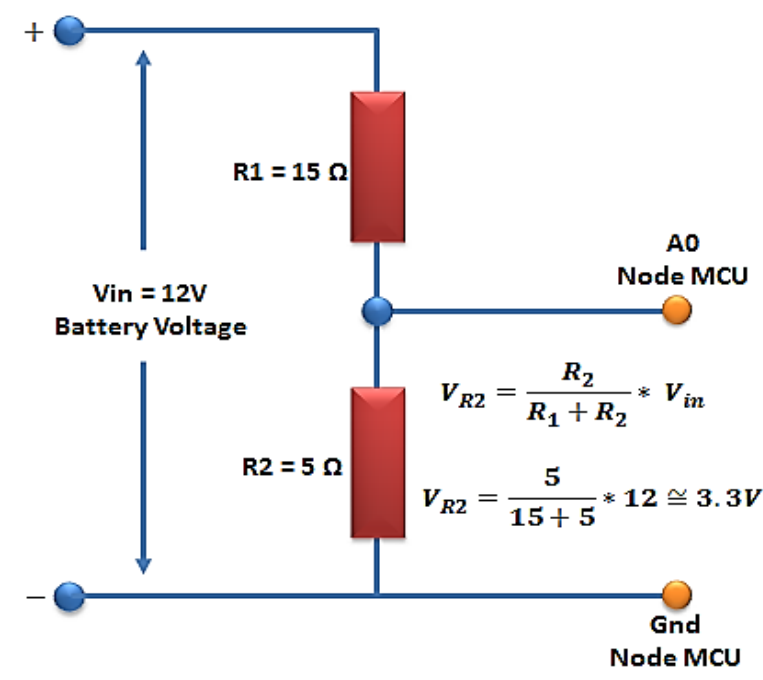

Fig.10. Voltage Divider Method (12 - 3.3) VDC 


\section{Conclusion}

This paper presents a creative, reliable and simple low-cost smart house system. The system can work under the supervision of IoT technology, which effectively automates environments remotely. The ingenuity of this innovative smart house system enables the web server-based house to be monitored and controlled via ESP8266 microcontroller. The outcomes of the employed sensors are intended to be displayed as semi charts (percentage gages) through NET PI webserver. The household tools can be switched (ON-OFF) in terms of network connectivity at any period and anywhere. Thanks to the total reliance on the webserver control system and the applicability of the local IP provided by the ESP8266 unit that leads to the cost constraints of the design to be inexpensive. NETPI is almost identical to other platforms except with a little of complexities in the programming manner. Also as engineers; we're still searching for ventures that work with the highest accuracy and the lowest price as NETPI platform. Finally, the restrictions to use only one node MCU module in a single web cloud application were violated and the implementation was approved uniquely in this work.

\section{References}

[1] Dhanalaxmi, B., \& Naidu, G. A. (2017, February). A survey on design and analysis of robust IoT architecture. In 2017 International Conference on Innovative Mechanisms for Industry Applications (ICIMIA) (pp. 375-378). IEEE.

[2] Patchava, V., Kandala, H. B., \& Babu, P. R. (2015, December). A smart home automation technique with raspberry pi using iot. In 2015 International Conference on Smart Sensors and Systems (IC-SSS) (pp. 1-4). IEEE.

[3] Medina, B. E., \& Manera, L. T. (2017, May). Retrofit of air conditioning systems through an wireless sensor and actuator network: An iot-based application for smart buildings. In 2017 IEEE 14th International Conference on Networking, Sensing and Control (ICNSC) (pp. 49-53). IEEE.

[4] Harsha, S. S., Reddy, S. C., \& Mary, S. P. (2017, February). Enhanced home automation system using internet of things. In 2017 International Conference on I-SMAC (IoT in Social, Mobile, Analytics and Cloud)(I-SMAC) (pp. 89-93). IEEE.

[5] Karthikeyan, S., \& Bhuvaneswari, P. T. V. (2017, January). Iot based real-time residential energy meter monitoring system. In 2017 Trends in Industrial Measurement and Automation (TIMA) (pp. 1-5). IEEE.

[6] Yacchirema, D. C., Palau, C. E., \& Esteve, M. (2017, January). Enable IoT interoperability in ambient assisted living: Active and healthy aging scenarios. In 2017 14th IEEE Annual Consumer Communications \& Networking Conference (CCNC) (pp. 53-58). IEEE.

[7] Pan, J., Jain, R., Paul, S., Vu, T., Saifullah, A., \& Sha, M. (2015). An internet of things framework for smart energy in buildings: designs, prototype, and experiments. IEEE Internet of Things Journal, 2(6), 527-537.

[8] Vijay, Rampeesa, Thotakura Sainag, and A. Vamsee Krishna. "Smart Home Wireless Automation Technology using Arduino based on IOT." IJECT Journal 8, no. 4 (2017).

[9] Aziz, D. A. Design of Smart House System based on Webserver Architecture Control. International Journal of Computer Applications, 975, 8887.

[10] Saputra, L. K. P., \& Lukito, Y. (2017, November). Implementation of air conditioning control system using REST protocol based on NodeMCU ESP8266. In 2017 International Conference on Smart Cities, Automation \& Intelligent Computing Systems (ICON-SONICS) (pp. 126-130). IEEE. 
[11] Laksono, P. W., Jauhari, W. A., Iftadi, I., Ayu, K. C., Pandu, B. I., Jamaluddin, A., \& Haijunowibowo, D. (2015, November). A system based on fuzzy logic approach to control humidity and temperature in fungus cultivation. In Proceedings of the Joint International Conference on Electric Vehicular Technology and Industrial, Mechanical, Electrical and Chemical Engineering (ICEVT \& IMECE) (pp. 344-347). IEEE.

[12] Mahmood, S. N. (2018). GSM Interaction Based Real Time Climate Change Monitoring Technique. kirkuk university journal for scientific studies, 13(2), 1-17.

[13] Mahmood, S. N., \& Hasan, F. F. (2017). Design of weather monitoring system using Arduino based database implementation. Journal of Multidisciplinary Engineering Science and Technology (JMEST), ISSN, 2458-9403.

[14] Nograles, H. A. H., Agbay, C. P. D., Flores, I. S. L., Manuel, A. L., \& Salonga, J. B. C. (2014, May). Low cost internet based wireless sensor network for air pollution monitoring using zigbee module. In 2014 Fourth International Conference on Digital Information and Communication Technology and its Applications (DICTAP) (pp. 310-314). IEEE.

[15] Machappa, T., Sasikala, M., \& Prasad, M. A. (2010). Design of Gas Sensor Setup and Study of Gas (LPG) Sensing Behavior of Conducting Polyaniline/Magnesium Chromate (MgCrO \$ $\{4\}$ \$) Composites. IEEE Sensors Journal, 10(4), 807-813.

[16] Mahmood, S. N., Ishak, A. J., \& Hussain, S. T. (2019). GSM based gas leak monitoring system. Periodicals of Engineering and Natural Sciences, 7(2), 670-678.

[17] Mahmood, S. N., Abdulabbas, A., Easa, H., \& Algburi, S. S. (2017, April). The battery characteristics impact on solar systems: Performance and cost. In 2017 International Conference on Current Research in Computer Science and Information Technology (ICCIT) (pp. 151-156). IEEE.

[18] Hussain, A. S. T., \& Mahmood, S. N. (2017, August). Hybrid power station act based on run off river. In 2017 IEEE 3rd International Conference on Engineering Technologies and Social Sciences (ICETSS) (pp. 1-7). IEEE.

[19] Ishak, A. J., \& Mahmood, S. N. (2019). Eye in hand robot arm based automated object grasping system. Periodicals of Engineering and Natural Sciences, 7(2), 555-566. 